\title{
FROM COMMON ENVELOPE TO PRE-CATACLYSMIC VARIABLES: AN OBSERVATIONAL TEST OF COMMON ENVELOPE EVOLUTION
}

\author{
M. J. SARNA \\ N. Copernicus Astronomical Center, Polish Academy of \\ Sciences, ul. Bartycka 18, 00-716 Warsaw, Poland
}

\section{Evolutionary scheme for cataclysmic variables}

The generally accepted evolutionary scenario for cataclysmic variables (CVs) is common envelope (CE) evolution (Iben \& Livio 1993) proposed by Paczyński (1976). The secondary spirals towards the giant's compact core converting orbital energy into kinetic energy of the giant's envelope and the envelope is ejected. The dynamics of the red dwarf and red giant envelope interaction have been studied by several groups (Livio \& Soker 1988; Taam $\&$ Bodenheimer 1991). After the ejection of the red giant envelope the post common envelope detached binaries (PCEBs) are formed. These can be divided into three groups:

- Hot subdwarf with a red dwarf star inside a planetary nebula.

- Hot subdwarf with a red dwarf star without a planetary nebula.

- Hot white dwarf with a red dwarf star without a planetary nebula.

Next, due to angular momentum loss by magnetic braking and/or gravitational radiation, the red dwarf component fills its Roche lobe and a cataclysmic variable is formed.

\section{Spherically symmetric accretion}

$\mathrm{CE}$ evolution is a very important factor in the structure of the secondary component in PCEBs. Let us first consider spherically symmetric accretion onto the main sequence star. It is a well known fact that a star with a radiative envelope or a thin convective zone $\left(M_{*}>0.55 M_{\odot}\right)$ expands during accretion (Kippenhahn \& Meyer-Hofmeister 1977). For a star which has a thick convective envelope, or is fully convective, the effectiveness of heating is crucial: 
- in the case where the entropy of the infalling matter is greater than that in the outer layer of the accreting star the reaction of the star is determined by the formation of a radiative temperature inversion layer below the stellar surface (Prialnik \& Livio 1985; Sarna \& Ziółkowski 1987). The radius of the star grows rapidly in response to accretion;

- in the case where the entropy of the infalling matter is less than or comparable to that of the matter in the outer layers of the accreting star, the star should shrink upon accretion (Webbink 1976; Whyte \& Eggleton 1985).

The response of an accreting main sequence star within a $\mathrm{CE}$ will be characterized by the first case, where entropy is higher.

\section{Accretion during common envelope evolution}

Hjellming \& Taam (1991) considered the response of a $1.25 \mathrm{M}_{\odot}$ main sequence star to the conditions within the envelope of a 2 or $5 \mathrm{M}_{\odot}$ red giant. There are two phases characterizing the evolution of a main sequence star inside the red giant's envelope: the mass accretion and the mass loss phases. During accretion, the original surface layers become nearly isothermal while the outer layers of accreted material have normal temperature gradients. Very high luminosities are produced in the outer layers which drive expansion, increasing the radius by more than a factor of 10 . The star fills its Roche lobe and mass loss begins. The calculation ends when the radius decreases to its initial value and the giant's envelope has been expelled. The net addition of secondary mass during the CE phase is minimal. Note that during the mass loss stage of the secondary star during the CE phase the separation and period of the system decrease. This happens because the mass of the carbon-oxygen core is less than that of the secondary star.

A quite different situation occurs for a lower mass secondary. For example, if we consider a $0.3 \mathrm{M}_{\odot}$ secondary, its response to accretion will be the same as for a $1.25 \mathrm{M}_{\odot}$ star, but its internal structure will be different (Prialnik \& Livio 1985; Sarna \& Ziółkowski 1987). After accretion of 0.1..0.2 $\mathrm{M}_{\odot}$ the radius will expand by a factor of a few and the secondary fills its Roche lobe and mass loss begins. But now mass loss (transfer) is unstable, as pointed out by Hameury et al. (1993). The separation and period at this stage start to increase, because now the mass of the carbon-oxygen core is greater than the mass of the secondary star. The extended secondary shrinks below its Roche lobe. We suggest that the net addition to the secondary's mass will not be negligible. 


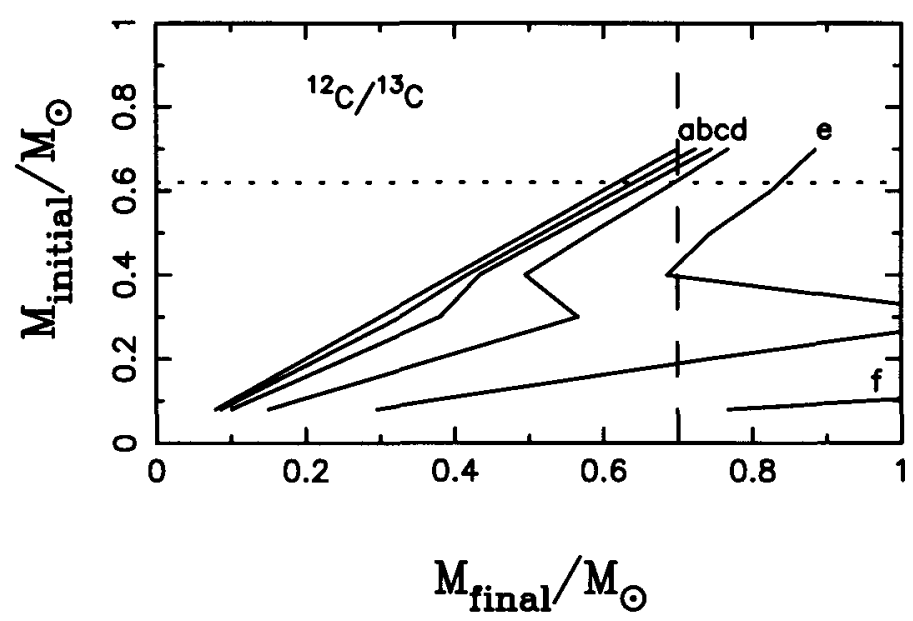

Figure 1. The ${ }^{12} \mathrm{C} /{ }^{13} \mathrm{C}$ ratio as a function of the pre-CE and PCEB red dwarf masses. The curves of constant isotopic ratio indicated by the letters $a, b, c, d$, e and $\mathbf{f}$ correspond to values of $91,70,50,30,20$ and 16 , respectively. The vertical, dashed, line is for a $0.7 \mathrm{M}_{\odot}$ red dwarf; if its isotopic ratio is 50 , then its initial mass was $0.62 \mathrm{M}_{\odot}$.

\section{Isotopic ratios for dwarf and giant stars}

It is a well known fact that the ratios of the various isotopes change during different phases of stellar evolution (Iben 1967; El Eid 1994). If the star evolves towards the red giant region a thick convective envelope develops changing the distribution of isotopes. Typical values (Harris, Lambert \& Smith 1988) of the ${ }^{12} \mathrm{C} /{ }^{13} \mathrm{C}$ ratio for main sequence stars and red giants are respectively 90 and $14 \pm 1.5$. The error for the red giant values is typical of determinations of the isotopic ratios for particular giants.

\section{Basic idea}

The basic assumptions are that during $\mathrm{CE}$ evolution the main sequence star accretes some amount of the giant's envelope, and after the CE phase, when the secondary relaxes to its thermal equilibrium configuration, the accreted material is mixed with the convective envelope of the red dwarf. The ${ }^{12} \mathrm{C} /{ }^{13} \mathrm{C}$ ratios after relaxation are shown in Fig. 1, where initial mass means the mass of the red dwarf before the common envelope phase, and final after (Sarna et al. 1995). If we have a mass determination for the red dwarf component in the PCEB, and also have an isotopic ratio determination for carbon, using this graph we can determine the initial mass of the red dwarf and hence the amount of mass which is accreted during the $\mathrm{CE}$ phase. 


\section{Infrared observation of cataclysmic variables}

We propose (Sarna et al. 1995) a simple observational test based on the infrared measurement of the ${ }^{12} \mathrm{C} /{ }^{13} \mathrm{C}$ ratio using the $\mathrm{CO}$ bands. Dhillon \& Marsh (1995) observed the $K$-band echelle spectra of the detached PCEB V471 Tau. The spectrum of V471 Tau shows ${ }^{12} \mathrm{CO}$ but this is rotationally broadened due to the $12.51 \mathrm{~h}$ orbital period, which smears the absorption profile. The ${ }^{12} \mathrm{CO}$ band in V471 Tau is also red-shifted due to the orbital motion. There is a marginal detection of ${ }^{13} \mathrm{CO}$ around 2.346 microns.

\section{Prediction}

The dwarf stars in PCEBs with planetary nebulae, in which the secondary is not in thermal equilibrium due to the short time since the CE phase, will exhibit ${ }^{12} \mathrm{C} /{ }^{13} \mathrm{C}$ ratios typical of giants. The best candidates are: V477 Lyr, UU Sge.

The dwarf stars in PCEBs without planetary nebulae, in which the secondary is in thermal equilibrium given the long time since the $\mathrm{CE}$ phase, should exhibit ${ }^{12} \mathrm{C} /{ }^{13} \mathrm{C}$ ratios intermediate between giants and solar values. The best candidates are Feige 24, HZ 9, V471 Tau.

\section{References}

Dhillon, V.S., Marsh, T.R., 1995, MNRAS, 275, 89

El Eid, M.F., 1994, A\&A, 285, 915

Hameury, J.-M., King, A.R., Lasota J.-P., Raison F., 1993, A\&A, 277, 81

Harris, M.L., Lambert, D.L., Smith, V.V., 1988, Ap. J., 325, 768

Hjellming, M.S., Taam, R.E., 1991, Ap. J., 370, 709

Iben, I., 1967, ARAA, 5, 571

Iben, I., Livio, M., 1993, PASP, 105, 1373

Kippenhahn, R., Meyer-Hofmeister, E., 1977, A\&A, 54, 539

Livio, M., Soker, N., 1988, Ap. J., 329, 764

Paczyński, B., 1976, in "The Structure and Evolution of Close Binary Systems", eds. P.P. Eggleton, S. Mitton, J. Whelan (Dordrecht, Reidel), p75

Prialnik, D., Livio, M., 1985, MNRAS, 216, 37

Sarna, M.J., Ziółkowski, J., 1987, Acta Astron., 38, 89

Sarna, M.J., Dhillon, V.S., Marsh, T.R., Marks, P.B., 1995, MNRAS, 272, 141

Taam, R.E., Bodenheimer, P., 1991, Ap. J., 373, 246

Webbink, R.F., 1976, Ap. J. Supp., 32, 583

Whyte, C., Eggleton, P.P., 1985, MNRAS, 214, 357 